\title{
Extensions of Fast-Lipschitz Optimization for Convex and Non-convex Problems ${ }^{\star}$
}

\author{
Martin Jakobsson and Carlo Fischione
}

\author{
KTH Royal Institute of Technology, Stockholm, Sweden \\ $\{$ mjakobss,carlofi\}@kth.se
}

\begin{abstract}
Fast-Lipschitz optimization has been recently proposed as a new framework with numerous computational advantages for both centralized and decentralized convex and nonconvex optimization problems. Such a framework generalizes the interference function optimization, which plays an essential role distributed radio power optimization over wireless networks. The characteristics of Fast-Lipschitz methods are low computational and coordination complexity compared to Lagrangian methods, with substantial benefits particularly for distributed optimization. These special properties of Fast-Lipschitz optimization can be ensured through qualifying conditions, which allow the Lagrange multipliers to be bound away from zero. In this paper, the Fast-Lipschitz optimization is substantially extended by establishing new qualifying conditions. The results are a generalization of the old qualifying conditions and a relaxation of the assumptions on problem structure so that the optimization framework can be applied to many more problems than previously possible. The new results are illustrated by a non-convex optimization problem, and by a radio power optimization problem which cannot be handled by the existing Fast-Lipschitz theory.
\end{abstract}

Keywords: Non-convex optimization, Distributed optimization, Wireless sensor networks, Radio power control

\section{INTRODUCTION}

Many networked optimization problems require that the nodes or agents in the network choose decision variables optimizing some global objective function. Examples are the minimization of the variance of some distributed estimation in a wireless sensor network, Fischione (2011), or the radio power control problem in cellular networks where the objective is to minimize the transmit powers with respect to some quality of service constraints, Boche and Schubert (2008). Due to the possible sheer size of the network and decision variables, or that most coordination information is only locally available at each node, it might be unpractical or even impossible to compute the optimal solution centrally. Instead, distributed algorithms must be used.

Most existing methods assume that the global objective can be split into a sum of convex functions, one belonging to each node. Dual decomposition and subgradient methods can then be applied, recent works are Nedic and Ozdaglar (2009); Rantzer (2009); Boyd (2010); Sundhar Ram et al. (2010). As these methods typically have slow rates of convergence, recent papers explores higher order methods, see Zanella et al. (2011); Wei et al. (2011). Other methods are based on the consensus iterations, where nodes update their decision variables as a weighted sum of other decision variables in the neighborhood, see, e.g., Notarstefano and Bullo (2009); Olshevsky and Tsitsiklis (2009); Nedic et al. (2010); Chiuso et al. (2011). However, calculating and updating the dual variables or

\footnotetext{
* This paper is supported by the EU projects HydroBioNets and Hycon2 and the Swedish Research Council.
}

making consensus steps requires some extra coordination and communication. Such an extra communication is always associated with increased cost, for example in time delays or energy consumption. This is a problem especially in wireless sensor networks or large scale distributed networks, where communication can be a hundred times more energy intensive than computation.

Fast-Lipschitz optimization is a new approach that circumvents the need of communicating Lagrangian multipliers or making consensus iterations. Thus, it substantially reduces the computational complexity for a class of optimization problems. The approach is applicable in any situation where the problem fulfills certain properties and to both convex and non-convex problems. In particular, one should be able to rewrite the problem as a monotonic maximization with one constraint for each decision variable. Certain qualifying conditions then give sufficient conditions for the existence of optimal solutions that are fixed points of the constraints, see Fischione (2011). However, such a theory is in its infancy, much still needs to be investigated.

In this paper, we substantially extend the class of problems that are currently solvable by the Fast-Lipschitz method. This is done by both generalizing the original qualifying conditions, and by relaxing some of the assumptions on the problem structure. The challenging part of the new analysis is based on showing that the Lagrange multipliers are bounded away from zero by the theory of contractions and matrix norms. Once the Lagrange multipliers are positive, the complementary slackness of the KKT conditions require that the optimal point is a fixed point of the constraints. When the constraints are contractive, 
the optimal point is unique and can be found by iterating the constraint functions.

\subsection{Notation}

Vectors are columns and denoted by lower bold case letters, matrices are denoted with upper bold case letters. The components of a vector $\mathbf{x}$ are denoted $x_{i}$ or $[\mathbf{x}]_{i}$. Similarly, the elements of the matrix $\mathbf{A}$ are denoted $A_{i j}$ or $[\mathbf{A}]_{i j}$. The transpose of a vector or matrix is denoted ${ }^{T}$. The gradient of a function should be interpreted as $[\nabla \mathbf{f}(\mathbf{x})]_{i j}=\partial f_{j} / \partial x_{i}, \nabla_{i} \mathbf{f}$ denotes the $i$-th row of $\nabla \mathbf{f} . \rho(\mathbf{A})$ denotes the spectral radius of $\mathbf{A},\|\cdot\|$ denotes any induced matrix norm and $\|\cdot\|_{\infty}$ is the norm induced from the $\ell_{\infty}$ vector norm $\left(\|\mathbf{A}\|_{\infty}=\max _{i} \sum_{j}\left|A_{i j}\right|\right)$. I and $\mathbf{1}$ denote the identity matrix and the vector $(1, \ldots, 1)^{T}$ respectively, and $\mathbf{0}$ denotes a zero vector or matrix. All inequalities are intended element-wise, i.e., they have nothing to do with positive definiteness.

\section{BACKGROUND WORK}

In this section, we recall an example of distributed nonconvex optimization that motivates the need of fast distributed solvers, and the definition of Fast-Lipschitz optimization.

\subsection{A Motivating Example}

Consider a wireless network where pairs of nodes are communicating. We model the network as a graph. In particular we consider a graph, $\mathcal{G}(t)=(\mathcal{V}, \mathcal{E})$, where $\mathcal{V}=\{1, \ldots, N\}$ is the nodes set and $\mathcal{E} \subseteq \mathcal{V} \times \mathcal{V}$ is the links set. Every link is associated only to a pair of nodes, one transmitting and one receiving some information.

Interference function optimization problems were first posed by Foschini and Miljanic (1993) and formalized by Yates (1995). They have the typical form

$$
\begin{aligned}
& \min \mathbf{p} \\
& \text { s.t. } p_{i} \geq \mathcal{I}_{i}(\mathbf{p}) \quad \forall i .
\end{aligned}
$$

The goal is to minimize the radio power $p_{i}$ used by every transmit node, while maintaining a minimum signal-tonoise ratio $\eta_{i}$ needed to successfully decode information at the receive node. One of the simplest examples considers the gain matrix $\mathbf{G}$, where $G_{i j}$ is the gain from transmitter $j$ to receiver $i$. The noise at receiver $i$ consists of the background noise $\sigma_{i}$ plus the interference of all other links, $\sum_{j \neq i} G_{i j} p_{j}$. This gives the requirement

$$
p_{i} \geq \mathcal{I}_{i}(\mathbf{p})=\frac{\eta_{i}}{G_{i i}}\left(\sum_{j \neq i} G_{i j} p_{j}+\sigma_{i}\right) \quad \forall i .
$$

A function $\mathcal{I}(\mathbf{p})$ is called standard if for all $\mathbf{p} \geq \mathbf{0}$

- Monotonicity: If $\mathbf{p} \geq \mathbf{p}^{\prime}$, then $\mathcal{I}(\mathbf{p}) \geq \mathcal{I}\left(\mathbf{p}^{\prime}\right)$.

- Scalability: For all $\alpha>1, \alpha \mathcal{I}(\mathbf{p})>\overline{\mathcal{I}}(\alpha \mathbf{p})$.

When problem (1) above is feasible, and the functions $\mathcal{I}_{i}(\mathbf{p})$ are standard, the unique solution is given by the fixed point of the iteration

$$
p_{i}^{k+1}=\mathcal{I}_{i}\left(\mathbf{p}^{k}\right),
$$

where $\mathbf{p}^{k}=\left[p_{1}^{k}, p_{2}^{k}, \ldots, p_{n}^{k}\right]$. The computation of the optimal solution by these iterations is much simpler than using Lagrangian multipliers and the classical parallelization and decomposition methods, Bertsekas and Tsitsiklis (1997), because no Lagrangian multipliers have to be somewhere centrally computed and redistributed to the nodes along with the decision variables. The iterations need only that every node keeps making them by using local knowledge of other nodes' current decision variables (radio powers). Another advantage is that converge is obtained even though such a knowledge is delayed, i.e., the decision variables $p_{j}^{k}$ of other nodes come with some delay at node $i$.

In Sung and Leung (2005), the authors consider an optimization problem where the interference functions are not standard by Yates' definition. Instead, they introduce Type-II standard functions, which fulfill:

- Type-II monotonicity: If $\mathbf{p} \leq \mathbf{p}^{\prime}$, then $\mathcal{I}(\mathbf{p}) \geq \mathcal{I}\left(\mathbf{p}^{\prime}\right)$.

- Type-II scalability: $\forall \alpha>1, \mathcal{I}(\alpha \mathbf{p})>(1 / \alpha) \mathcal{I}(\mathbf{p})$.

These functions are shown to have the same fixed point properties as Yates' standard functions, i.e., problem (1) with Type-II standard constraints can be solved through repeated iterations of the constraints.

A further extension of the interference function framework has been proposed in Boche and Schubert (2008). The generalization consists in replacing Yates' assumption of scalability with

- Scale invariance: $\forall \alpha>0, \mathcal{I}(\alpha \mathbf{p})=\alpha \mathcal{I}(\mathbf{p})$.

A natural question that arises is how to solve distributed optimization problems over networks by using fixed point iterations similar to (2), but when the constraints do not satisfy the standard or Type-II or Boche's conditions. This motivates Fast-Lipschitz optimization.

\subsection{Fast-Lipschitz Optimization}

A recent paper, Fischione (2011), has proposed the new framework of Fast-Lipschitz optimization. It considers a class of possibly non-convex and multi-objective problems with monotonic objective functions. These problems have unique Pareto optimal solutions, well defined by a contractive system of equations involving the problem constraints. Therefore, Fast-Lipschitz problems can be solved without having to introduce Lagrangian functions and dual variables. This makes the framework particularly well suited when highly decentralized solutions are required with few coordination messages, such as for example in wireless sensor networks and in multi-agent systems.

We will now give a formal definition (slightly different from the original article) of Fast-Lipschitz problems. For a thorough discussion of Fast-Lipschitz properties we refer to the above mentioned paper.

Definition 1. A problem is said to be on Fast-Lipschitz form if it can be written

$$
\begin{array}{cl}
\max & \mathbf{f}_{0}(\mathbf{x}) \\
\text { s.t. } & x_{i} \leq f_{i}(\mathbf{x}) \quad \forall i \in \mathcal{I} \\
& x_{i}=f_{i}(\mathbf{x}) \quad \forall i \in \mathcal{E} \\
& \mathbf{x} \in \mathcal{D} \subset \Re^{n}
\end{array}
$$

Here, $\mathbf{f}_{0}$ is a possibly vector valued function $\mathbf{f}_{0}: \mathcal{D} \subset$ $\Re^{n} \rightarrow \Re^{m}, \quad m \geq 1$. $\mathcal{I}$ and $\mathcal{E}$ are complementary subsets 
of $\{1, \ldots, n\}$ and for each $f_{i}$ can be collected as the $i$-th component of the vector valued function $\mathbf{f}: \mathcal{D} \subset \Re^{n} \rightarrow$ $\Re^{n}$, i.e., $\mathbf{f}(\mathbf{x})=\left[f_{1}(\mathbf{x}) \cdots f_{n}(\mathbf{x})\right]^{T}$. The last constraint is a box constraint, meaning $\mathcal{D}=\left\{\mathbf{x} \in \Re^{n} \mid \mathbf{a} \leq \mathbf{x} \leq \mathbf{b}\right\}$. D does not need to cover the entire feasible region determined by the two previous constraints, but $\mathcal{D}$ must contain the point $\mathbf{x}^{\star}$ where all equalities hold strictly, i.e., $\mathcal{D} \ni \mathbf{x}^{\star}=$ $\mathbf{f}\left(\mathbf{x}^{\star}\right)$.

Remark 2. The characteristic feature of Fast-Lipschitz form is that each variable has exactly one constraint (apart from the box $\mathcal{D}$ ). The form $x \leq f(\mathbf{x})$ is general, since any constraint on canonical form, $g(\mathbf{x}) \leq 0$, can be written $x \leq x-\gamma g(\mathbf{x})$ for some positive constant $\gamma$.

Definition 3. A problem is said Fast-Lipschitz when it can be written on Fast-Lipschitz form and, if feasible, admits a unique Pareto optimal solution $\mathbf{x}^{\star}$, defined as the unique solution to the system of equations

$$
\mathbf{x}^{\star}=\mathbf{f}\left(\mathbf{x}^{\star}\right) .
$$

A problem on Fast-Lipschitz form is not automatically Fast-Lipschitz. Conditions that, together with FastLipschitz form, guarantee that a problem is Fast-Lipschitz are called qualifying conditions.

Qualifying Conditions 1. For all $\mathbf{x}$ in $\mathcal{D}, \mathbf{f}_{0}(\mathbf{x})$ and $\mathbf{f}(\mathbf{x})$ should be continuously differentiable and fulfill at least one of the following cases:

$$
\text { (0) } \nabla \mathbf{f}_{0}(\mathbf{x})>\mathbf{0}
$$

and

$$
\begin{aligned}
& \text { (i.a) } \nabla \mathbf{f}(\mathbf{x}) \geq \mathbf{0} \\
& \text { (i.b) }\|\nabla \mathbf{f}(\mathbf{x})\|<1 \text { for some norm }\|\cdot\|
\end{aligned}
$$

or

$$
\begin{aligned}
& \text { (ii.a) } \nabla_{i} \mathbf{f}_{0}(\mathbf{x})=\nabla_{j} \mathbf{f}_{0}(\mathbf{x}) \quad \forall i, j \\
& \text { (ii.b) } \nabla \mathbf{f}(\mathbf{x}) \leq \mathbf{0}\left(\text { or } \nabla \mathbf{f}(\mathbf{x})^{2} \geq \mathbf{0}\right) \\
& \text { (ii.c) }\|\nabla \mathbf{f}(\mathbf{x})\|_{\infty}<1 \\
& \quad \text { or }
\end{aligned}
$$

$$
\begin{aligned}
& \text { (iii.a) } \mathbf{f}_{0}(\mathbf{x})>\mathbf{0} \in \Re \\
& \text { (iii.b) }\|\nabla \mathbf{f}(\mathbf{x})\|_{\infty}<\frac{\delta}{\delta+\Delta}
\end{aligned}
$$

where $\delta=\min _{i, \mathbf{x}} \nabla_{i} \mathbf{f}_{0}(\mathbf{x})$ and $\Delta=\max _{i, \mathbf{x}} \nabla_{i} \mathbf{f}_{0}(\mathbf{x})$.

Theorem 4. (Fischione, 2011, Theorem 3.3) A problem on Fast-Lipschitz form that fulfills Qualifying Condition 1 is Fast-Lipschitz, i.e., it has a unique Pareto optimal point given by $x^{\star}=\mathbf{f}\left(x^{\star}\right)$.

Once it is known that a problem is Fast-Lipschitz, computing the solution becomes a matter of solving the system of equations (4), which in general is much easier than solving an optimization problem using Lagrangian multipliers. This is particularly true when $\mathbf{f}(\mathbf{x})$ is contractive on $\mathcal{D}$, a property assured by Qualifying Condition 1 . Then, the iterations $\mathbf{x}^{k+1}:=\mathbf{f}\left(\mathbf{x}^{k}\right)$ converge geometrically to the optimal point $\mathbf{x}^{\star}$, starting from any initial point $\mathbf{x}^{0} \in \mathcal{D}$.

Fast-Lipschitz optimization problems need not be convex, but many convex problems can be rewritten and solved with the Fast-Lipschitz framework (provided that they fulfill the qualifying conditions). This is true for any problem being either standard or type-II standard:

Proposition 5. If the constraints of problem (1) are either standard or type-II standard, then the problem is FastLipschitz.

In the following section, we provide a substantial extension of Fast-Lipschitz optimization.

\section{NEW QUALIFYING CONDITIONS}

This section is the core contribution of the paper and presents a novel set of qualifying conditions, greatly extending the earlier Qualifying Conditions 1.

Consider once again optimization problem (3), but with $\mathbf{f}(\mathbf{x})$ and $\mathbf{f}_{0}(\mathbf{x})$ fulfilling the following conditions:

Qualifying Conditions 2. Let $\delta(\mathbf{x})=\min _{i, j}\left[\nabla \mathbf{f}_{0}(\mathbf{x})\right]_{i j}$ and $\Delta(\mathbf{x})=\max _{i, j}\left[\nabla \mathbf{f}_{0}(\mathbf{x})\right]_{i j}$. For all $\mathbf{x}$ in $\mathcal{D}$, the following conditions must hold:

(0) $\nabla \mathbf{f}_{0}(\mathbf{x}) \geq \mathbf{0}$ with non-zero rows

and

$$
\begin{aligned}
& \text { (i.a) } \nabla \mathbf{f}(\mathbf{x}) \geq \mathbf{0} \\
& \text { (i.b) }\|\nabla \mathbf{f}(\mathbf{x})\|<1 \text { for some norm }\|\cdot\| \\
& \quad \text { or } \\
& \text { (ii.a) } \nabla \mathbf{f}(\mathbf{x})^{2} \geq \mathbf{0}, \text { e.g., } \nabla \mathbf{f}(\mathbf{x}) \leq \mathbf{0} \\
& \text { (ii.b) }\|\nabla \mathbf{f}(\mathbf{x})\|_{\infty}<\frac{\delta(\mathbf{x})}{\Delta(\mathbf{x})}
\end{aligned}
$$$$
\text { or }
$$$$
\text { (iii.a) }\|\nabla \mathbf{f}(\mathbf{x})\|_{\infty}<\frac{\delta(\mathbf{x})}{\delta(\mathbf{x})+\Delta(\mathbf{x})}
$$

Case (ii) and case (iii) of the conditions above are highly related. In fact, they are both special cases of a more general case:

There exists an integer $k \geq 1$ such that

$$
\begin{aligned}
& \text { (iv.a) }(\nabla \mathbf{f}(\mathbf{x}))^{k} \geq \mathbf{0}, \quad k=2,3, \ldots \\
& \left(\text { iv.b) }\left\|\sum_{l=1}^{k-1} \nabla \mathbf{f}(\mathbf{x})^{l}\right\|_{\infty}<\frac{\delta(\mathbf{x})}{\Delta(\mathbf{x})} .\right.
\end{aligned}
$$

Qualifying Conditions 1 hold as a special case of Qualifying Conditions 2. For example, when $\nabla_{i} \mathbf{f}_{0}=\nabla_{j} \mathbf{f}_{0}$ for any $i$ and $j, \delta=\Delta$ wherefore condition (ii.b) again requires $\|\nabla \mathbf{f}(\mathbf{x})\|_{\infty}<1$. Case (iii) can now also handle vector valued objective functions, and allows any problem on Fast-Lipschitz form to be Fast-Lipschitz if the constraint functions rate of change are sufficiently low.

Theorem 6. When problem (3) is feasible, with $\mathbf{f}_{0}$ and $\mathbf{f}$ fulfilling Qualifying Conditions 2, the problem is FastLipschitz, i.e., the unique Pareto optimal solution is given by $\mathbf{x}^{\star}=\mathbf{f}\left(\mathbf{x}^{\star}\right)$.

Proof. For simplicity, the proof will assume that all the constraints of the problem are inequalities. This is without loss of generality, since replacing equalities with inequalities relaxes the problem, while the optimal solution still has all constraints holding with equality. 
First, the vector optimization problem is scalarized by introducing the vector $\boldsymbol{\mu}>\mathbf{0}$, which is normalized such that $\sum \mu_{i}=1$ :

$$
\begin{array}{cl}
\max & \boldsymbol{\mu}^{T} \mathbf{f}_{0}(\mathbf{x}) \\
\text { s.t. } & \mathbf{x}_{i} \leq \mathbf{f}_{i}(\mathbf{x}) \quad 1, \ldots, n
\end{array}
$$

By varying $\boldsymbol{\mu}$, one can obtain all Pareto optimal solutions. The proof will show that the optimal point is not influenced by $\boldsymbol{\mu}$, i.e., there is a unique Pareto optimum.

The Lagrangian function is

$$
L(\mathbf{x}, \boldsymbol{\lambda})=\boldsymbol{\mu}^{T} \mathbf{f}_{0}(\mathbf{x})-\boldsymbol{\lambda}^{T}(\mathbf{x}-\mathbf{f}(\mathbf{x})) .
$$

Any pair $(\hat{\mathbf{x}}, \hat{\boldsymbol{\lambda}})$ of candidates for optimal variables must satisfy the KKT-conditions. In particular, $\hat{\mathbf{x}}$ must be the minimizer of $L(\mathbf{x}, \hat{\boldsymbol{\lambda}})$ which requires

$$
\nabla_{x} L(\hat{\mathbf{x}}, \hat{\boldsymbol{\lambda}})=\mathbf{0}
$$

and strict complementarity must hold, i.e.,

$$
\sum_{i=1}^{n} \hat{\lambda}_{i}\left(\hat{x}_{i}-f_{i}(\hat{\mathbf{x}})\right)=\hat{\boldsymbol{\lambda}}^{T}(\hat{\mathbf{x}}-\mathbf{f}(\hat{\mathbf{x}}))=0 .
$$

The idea of the proof is showing that $\hat{\lambda}_{i}>0$ for all $i$, wherefore (8) requires $\hat{\mathbf{x}}=\mathbf{f}(\hat{\mathbf{x}})$. In other words, any candidate for optimality is a fixed point of $\mathbf{f}(\mathbf{x})$. As $\|\nabla \mathbf{f}(\mathbf{x})\|<1$ for all $\mathbf{x}$ in $\mathcal{D}, \mathbf{f}(\mathbf{x})$ is a contraction on $\mathcal{D}$ and by the Banach Fixed Point Theorem, the fixed (optimal) point $\hat{\mathbf{x}}=\mathbf{x}^{\star}=\mathbf{f}\left(\mathbf{x}^{\star}\right)$ is unique.

From (7) we have

$$
\nabla_{x} L(\hat{\mathbf{x}}, \hat{\boldsymbol{\lambda}})=\nabla \mathbf{f}_{0}(\hat{\mathbf{x}}) \boldsymbol{\mu}-\hat{\boldsymbol{\lambda}}+\nabla \mathbf{f}(\hat{\mathbf{x}}) \hat{\boldsymbol{\lambda}}=\mathbf{0} .
$$

For a simpler notation we introduce

$$
\mathbf{c}=\nabla \mathbf{f}_{0}(\hat{\mathbf{x}}) \boldsymbol{\mu} \quad \text { and } \quad \mathbf{A}=\nabla \mathbf{f}(\hat{\mathbf{x}}) .
$$

To complete the proof, one must show how the properties of $\mathbf{A}$ and $\mathbf{c}$ imply $\hat{\boldsymbol{\lambda}}>\mathbf{0}$. As any candidate for optimality must be feasible, $\hat{\mathbf{x}}$ belongs to $\mathcal{D}$ and the required properties are obtained almost directly from Condition 2. Note that condition (0) gives $\mathbf{c}>\mathbf{0}$ for every $\boldsymbol{\mu}>\mathbf{0}$.

With the new notation, equation (9) can be written as

$$
\hat{\boldsymbol{\lambda}}=\mathbf{A} \hat{\boldsymbol{\lambda}}+\mathbf{c}, \text { or } \hat{\boldsymbol{\lambda}}=(\mathbf{I}-\mathbf{A})^{-1} \mathbf{c} .
$$

Assuming $\rho(\mathbf{A})<1,(\mathbf{I}-\mathbf{A})$ is invertible and (10) can be written as

$$
\hat{\boldsymbol{\lambda}}=\left(\mathbf{I}+\mathbf{A}+\mathbf{A}^{2}+\ldots\right) \mathbf{c}
$$

When case (0) and (i) holds, $\rho(\mathbf{A})<1, \mathbf{A} \geq \mathbf{0}$ and $\mathbf{c}>\mathbf{0}$ wherefore $\hat{\boldsymbol{\lambda}}>\mathbf{0}$.

For case (iv), factorize (11) as

$$
\begin{aligned}
\hat{\boldsymbol{\lambda}} & =\left(\mathbf{I}+\mathbf{A}^{k}+\mathbf{A}^{2 k}+\ldots\right)\left(\mathbf{I}+\mathbf{A}+\cdots+\mathbf{A}^{k-1}\right) \mathbf{c} \\
& =\sum_{l=0}^{\infty}\left(\mathbf{A}^{k}\right)^{l}\left(\mathbf{I}+\mathbf{A}+\cdots+\mathbf{A}^{k-1}\right) \mathbf{c} .
\end{aligned}
$$

Because of condition (iv.a) $\left(\nabla \mathbf{f}(\mathbf{x})^{k} \geq \mathbf{0}\right.$ for all $\mathbf{x}$ in $\left.\mathcal{D}\right)$, $\sum_{l=0}^{\infty}\left(\mathbf{A}^{k}\right)^{l}$ is non-negative with non-zero rows, so $\hat{\boldsymbol{\lambda}}>\mathbf{0}$ if $\left(\mathbf{I}+\mathbf{A}+\cdots+\mathbf{A}^{k-1}\right) \mathbf{c}>\mathbf{0}$, or equivalently, if

$$
-\left(\mathbf{A}+\cdots+\mathbf{A}^{k-1}\right) \mathbf{c} \equiv-\mathbf{B} \mathbf{c} \leq \mathbf{c}
$$

Since $\mathbf{c}>\mathbf{0}$, it is sufficient that the inequality above holds in absolute value. Considering the $i$-th row:

$$
\left|[-\mathbf{B c}]_{i}\right|=\mid \sum_{j=1}^{n}\left[\mathbf{B}_{i j} \mathbf{c}_{j}|\leq| \mathbf{c}_{i} \mid\right.
$$

By the definitions of $\mathbf{c}$ and $\delta$, we have

$$
\left|\mathbf{c}_{i}\right| \geq \min _{i} \mathbf{c}_{i}=\min _{i} \sum_{j=1}^{n}\left[\nabla \mathbf{f}_{0}(\hat{\mathbf{x}})\right]_{i j} \boldsymbol{\mu}_{j} \geq \sum_{j=1}^{n} \delta \boldsymbol{\mu}_{j}=\delta,
$$

since $\boldsymbol{\mu}>\mathbf{0}$ and $\sum_{j} \boldsymbol{\mu}_{j}=1$. Similarly, $\left|\mathbf{c}_{i}\right| \leq \Delta$.

Now, condition (iv.b) ensures

$$
\|\mathbf{B}\|_{\infty}=\left\|\sum_{l=1}^{k-1} \mathbf{A}^{l}\right\|_{\infty} \leq \frac{\delta}{\Delta}
$$

so we can write

$$
\left|\sum_{j=1}^{n} \mathbf{B}_{i j} \mathbf{c}_{j}\right| \leq \sum_{j=1}^{n}\left|\mathbf{B}_{i j}\right| \Delta=\|\mathbf{B}\|_{\infty} \Delta \leq \delta \leq \mathbf{c}_{i} .
$$

This proves case (iv). Case (ii) is obtained by setting $k=2$. For case (iii), let $k \rightarrow \infty$. Condition (iv.a) is then automatically fulfilled when $\|\nabla \mathbf{f}(\mathbf{x})\|<1$, since $(\nabla \mathbf{f})^{k} \rightarrow 0$ as $k \rightarrow \infty$. For condition (iv.b), write

$$
\begin{aligned}
\left\|\sum_{l=1}^{\infty} \mathbf{A}^{l}\right\|_{\infty} & =\left\|\mathbf{A} \sum_{l=0}^{\infty} \mathbf{A}^{l}\right\|_{\infty} \\
& \leq\|\mathbf{A}\|_{\infty} \sum_{l=0}^{\infty}\|\mathbf{A}\|_{\infty}^{l}=\frac{\|\mathbf{A}\|_{\infty}}{1-\|\mathbf{A}\|_{\infty}} .
\end{aligned}
$$

The inequality follows from the triangle inequality and the sub-multiplicativity of induced matrix norms. The geometric series converges since $\|\mathbf{A}\|_{\infty}<1$. Finally, the fraction above is less than $\delta / \Delta$ if $\|\mathbf{A}\|_{\infty}<\delta /(\delta+\Delta)$.

It should be noted that the conditions above are only sufficient. For example, by considering certain transformations of the constraint functions, it is possible to relax conditions concerning the norm $\|\cdot\|_{\infty}$. One can also relax certain requirements on the form of problem (3). Results for problems with more variables than constraints and problems where all variables does not appear in the cost function also exist, but are left out due to space limitations.

In the following section, we give some illustrative examples for both convex and non-convex optimization.

\section{EXAMPLES}

\subsection{Simple example}

Consider the problem

$$
\begin{aligned}
\max & \mathbf{f}_{0}(\mathbf{x}) \\
\text { s.t. } & \mathbf{x} \leq \mathbf{f}(\mathbf{x}) \\
& \mathbf{x} \in \mathcal{D}=\{\mathbf{x}: \mathbf{0} \leq \mathbf{x} \leq \mathbf{1}\}
\end{aligned}
$$

where $\mathbf{x} \in \mathbf{R}^{2}$,

$$
\mathbf{f}_{0}(\mathbf{x})=\left[\begin{array}{l}
2 x_{1}+x_{2} \\
x_{1}+2 x_{2}
\end{array}\right], \quad \text { and } \quad \mathbf{f}(\mathbf{x})=0.5\left[\begin{array}{c}
1+a x_{2}^{2} \\
1+b x_{1}^{2}
\end{array}\right] .
$$

If either $a$ or $b$ are positive, the problem is not convex (the canonical constraint functions $x_{i}-f_{i}(\mathbf{x})$ become concave). The gradients of the cost and constraint functions are

$$
\nabla \mathbf{f}_{0}=\left[\begin{array}{ll}
2 & 1 \\
1 & 2
\end{array}\right] \text { and } \nabla \mathbf{f}=\left[\begin{array}{cc}
0 & b x_{1} \\
a x_{2} & 0
\end{array}\right]
$$

Since $\nabla \mathbf{f}_{0}>\mathbf{0}$ for all $\mathbf{x}$, case (0) is always fulfilled. 
Case (i): If both $a$ and $b$ are non-negative, $\nabla \mathbf{f}(\mathbf{x}) \geq \mathbf{0}$ for all $\mathbf{x}$ in $\mathcal{D}$ and condition (i.a) holds. To verify condition (i.b), one must find a norm $\|\cdot\|$ such that $\|\nabla \mathbf{f}(\mathbf{x})\|<1$ for all $\mathbf{x}$ in $\mathcal{D}$. When the $\infty$-norm is used, we get

$\max _{\mathbf{x} \in \mathcal{D}}\|\nabla \mathbf{f}(\mathbf{x})\|_{\infty}=\max _{\mathbf{x} \in \mathcal{D}} \max \left\{\left|b x_{1}\right|,\left|a x_{2}\right|\right\} \leq \max \{b, a\}<1$ if $a, b<1$. Thus, when $0 \leq a, b<1$, the problem is FastLipschitz by cases $(\mathbf{0})+(\mathbf{i})$.

Case (ii): If $a$ and $b$ are instead non-positive, we have $\nabla \mathbf{f}(\mathbf{x}) \leq \mathbf{0}$ for all $\mathbf{x}$ in $\mathcal{D}$ and condition (ii.a) is fulfilled. In order to verify (ii.b), we need $\delta(\mathbf{x})$ and $\Delta(\mathbf{x})$. These are, defined pointwise in $\mathbf{x}$, as the smallest and largest (in absolute value) element of $\nabla \mathbf{f}_{0}$, i.e.,

$$
\delta(\mathbf{x})=1 \quad \text { and } \quad \Delta(\mathbf{x})=2 \quad \forall \mathbf{x} .
$$

Condition (ii.b) now requires, for all $\mathbf{x}$ in $\mathcal{D}$, that

$$
\|\nabla \mathbf{f}(\mathbf{x})\|_{\infty}<\frac{\delta(\mathbf{x})}{\Delta(\mathbf{x})}=\frac{1}{2}
$$

From the previous case we know that $\|\nabla \mathbf{f}(\mathbf{x})\|_{\infty} \leq$ $\max \{|a|,|b|\}$ for all $\mathbf{x} \in \mathcal{D}$, so the problem is guaranteed Fast-Lipschitz by cases (0)+(ii), provided that $-1 / 2<$ $a, b \leq 0$. Note that this would not have met the old condition (5d), since the rows of $\nabla \mathbf{f}_{0}$ are not identical.

Case (iii): When $a$ and $b$ have different signs, neither (i.a), nor (ii.a) holds. Instead, one can use case (iii), which does not place any sign restrictions on $\nabla \mathbf{f}(\mathbf{x})$. It is only required, in (iii.a), that

$$
\|\nabla \mathbf{f}(\mathbf{x})\|_{\infty}<\frac{\delta(\mathbf{x})}{\delta(\mathbf{x})+\Delta(\mathbf{x})} .
$$

These quantities are unchanged from the previous cases, so $\delta(\mathbf{x})=1, \Delta(\mathbf{x})=2$ and $\|\nabla \mathbf{f}(\mathbf{x})\|_{\infty} \leq \max \{|a|,|b|\}$, so the problem is Fast-Lipschitz by cases (0)+(iii) if both $|a|$ and $|b|$ are less than $1 / 3$.

Also in this case, the old qualifying conditions would not have worked since $(5 \mathrm{~g})$ requires a scalar cost function.

Solution of the problem If the problem is shown to be Fast-Lipschitz by any of the cases above, the optimal point $\mathbf{x}^{\star}$ is found by solving $\mathbf{x}^{\star}=\mathbf{f}\left(\mathbf{x}^{\star}\right)$. We now solve the problem when $a=-0.3$ and $b=0.3$ (case (iii)) by iterating

$$
\mathbf{x}^{k+1}=\mathbf{f}\left(\mathbf{x}^{k}\right)
$$

which will converge to $\mathbf{x}^{\star}=\mathbf{f}\left(\mathbf{x}^{\star}\right)$ since the qualifying conditions imply that $\mathbf{f}$ is contractive $(\|\nabla \mathbf{f}\|<1)$. The iterates $\mathbf{x}^{k}$, together with the feasible region of the problem is shown in Fig. 1. The convergence of the iterates is shown in Fig. 2.

\subsection{Interference function example}

Consider power problem (1), but let the interference of the $i$-th link be given by

$$
\begin{aligned}
\mathcal{I}_{i}(\mathbf{p}) & =\frac{\eta_{i}}{G_{i i}}\left(\sigma_{i}+\sum_{j \neq i} G_{i j} p_{j}+\sum_{j \neq i} M_{i j} p_{i}^{2} p_{j}^{2}\right) \\
& =\bar{\sigma}_{i}+\sum_{j \neq i} \bar{G}_{i j} p_{j}+\sum_{j \neq i} \bar{M}_{i j} p_{i}^{2} p_{j}^{2} .
\end{aligned}
$$

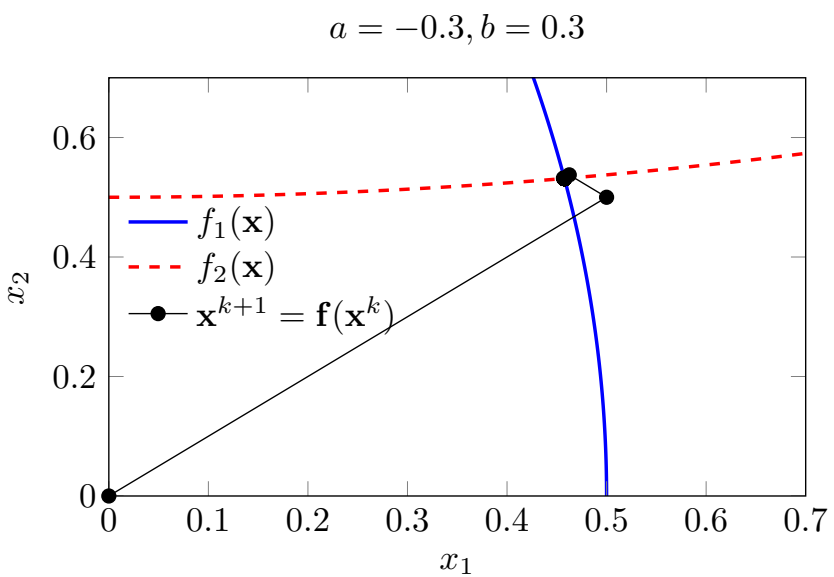

Fig. 1. Illustration of the feasible region with $a=-0.3$ and $b=0.3$ (case (iii)). The iterates of the solution quickly converges to $\mathbf{x}^{\star}=\mathbf{f}\left(\mathbf{x}^{\star}\right)$, where all constraints are active.

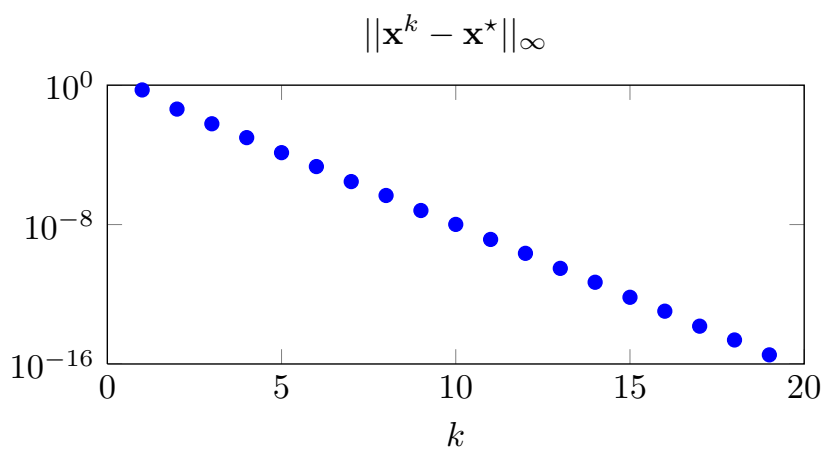

Fig. 2. Convergence of the iterates, measured in the $\infty$-norm. The convergence is geometric (linear in the log domain) and an accuracy of $10^{-6}$ is reached after 8 iterations.

The last sum models the intermodulation between link $i$ and $j$ (Boyd et al. (2007)). Assume also that the link powers are bound $\mathbf{0} \leq \mathbf{p} \leq \mathbf{p}_{\max }$. The constants $M_{i j}$ are generally small, but can be either positive or negative. In general, this new interference function is neither standard, nor Type-II standard, nor Boche's interference function. To see this, recall that monotonicity, and Type-II monotonicity requires $\nabla \mathcal{I}(\mathbf{p}) \geq \mathbf{0}$ and $\nabla \mathcal{I}(\mathbf{p}) \leq \mathbf{0}$ respectively. In this case, the gradient of $\mathcal{I}_{i}$ is

$$
\nabla_{j} \mathcal{I}_{i}(\mathbf{p})= \begin{cases}\bar{G}_{i j}+2 p_{i}^{2} \bar{M}_{i j} p_{j} & j \neq i \\ 2 p_{i} \sum_{k \neq i} \bar{M}_{i k} p_{k}^{2} & j=i\end{cases}
$$

A reasonable assumption is that the linear term dominates the intermodulation terms, wherefore the non-diagonal terms are positive. The diagonal terms can be negative if $\sum_{k \neq i} M_{i k}<0$, and in this case the terms of $\nabla \mathcal{I}$ are of mixed signs.

By introducing $\mathbf{x}=-\mathbf{p}$, the problem can be rewritten as

$$
\begin{aligned}
& \max \mathbf{f}_{0}(\mathbf{x})=\mathbf{x} \\
& \text { s.t. } x_{i} \leq f_{i}(\mathbf{x})=-\bar{\sigma}_{i}+\sum_{k \neq i}\left(\bar{G}_{i k} x_{k}-\bar{M}_{i k} x_{i}^{2} x_{k}^{2}\right) \quad \forall i \\
& \quad \mathbf{x} \in \mathcal{D}=\left\{\mathbf{x}:-\mathbf{p}_{\max } \leq \mathbf{x} \leq \mathbf{0}\right\}
\end{aligned}
$$


The mixed signs remain in $\nabla \mathbf{f}(\mathbf{x})$, wherefore neither case (i), nor case (ii) of Qualifying Condition 2 can be used. However, the simple form of $\mathbf{f}_{0}$ gives $\delta(\mathbf{x})=\Delta(\mathbf{x})=$ 1 , wherefore the problem qualifies through case (iii) if $\|\nabla \mathbf{f}(\mathbf{x})\|<1 / 2$ for all $\mathbf{x}$ in $\mathcal{D}$. We have

$$
\begin{aligned}
\|\nabla \mathbf{f}(\mathbf{x})\|_{\infty}=\max _{i} \sum_{j}\left|\nabla_{i} f_{j}(\mathbf{x})\right| \\
=\max _{i}\left\{\sum_{j \neq i}\left|\bar{G}_{i j}-2 \bar{M}_{i j} x_{i}^{2} x_{j}\right|\right. \\
\left.\quad+\left|-2 x_{i} \sum_{k \neq i} \bar{M}_{i k} x_{k}^{2}\right|\right\} .
\end{aligned}
$$

With the assumption that the interference is dominated by the linear terms, the absolute value can be removed from the terms in the first sum, and (remember $\mathbf{x} \leq \mathbf{0}$ )

$$
\begin{gathered}
\|\nabla \mathbf{f}(\mathbf{x})\|_{\infty}=\max _{i}\left\{\sum_{j \neq i}\left(\bar{G}_{i j}-2 \bar{M}_{i j} x_{i}^{2} x_{j}\right)\right. \\
\left.\quad+\sum_{j \neq i}\left|-2 x_{i}\right|\left|\bar{M}_{i j} x_{j}^{2}\right|\right\} \\
=\max _{i} \sum_{j \neq i}\left(\bar{G}_{i j}-2 x_{i}\left(\bar{M}_{i j} x_{i} x_{j}+\left|\bar{M}_{i j}\right| x_{j}^{2}\right)\right),
\end{gathered}
$$

whereby the problem is Fast-Lipschitz by the new results of this paper if

$$
\sum_{j \neq i}\left(\bar{G}_{i j}-2 x_{i}\left(\bar{M}_{i j} x_{i} x_{j}+\left|\bar{M}_{i j}\right| x_{j}^{2}\right)\right)<1 / 2
$$

for all $i$. The solution can then be found through repeated iterations of the constraint function, $\mathbf{x}^{k+1}=\mathbf{f}\left(\mathbf{x}^{k}\right)$. It can be shown that with problem parameters chosen to replicate a network of 10 Tmote Sky nodes, Fischione (2011), this method convergence in just 10 iterations whereas a traditional Lagrangian method requires 40 iterations.

\section{CONCLUSIONS AND FUTURE WORK}

In this paper we extended significantly previous FastLipschitz qualifying conditions. Based on these new results, many convex and non-convex optimization problems can be solved by the Fast-Lipschitz method proposed in this paper, both in a centralized and in a distributed set-up. This avoid using Lagrangian methods, which are inefficient in terms of computations and communication complexity when used over networks.

Several possible extensions remain to consider, for example by formulating the qualifying conditions in terms of diagonal dominance instead of infinity-norms. So far, only problems where $\nabla \mathbf{f}_{0} \geq \mathbf{0}$ have been considered. The standard inequality we have used is a partial ordering induced by the positive orthant $\Re_{+}^{n}$, i.e., $\nabla \mathbf{f}_{0} \geq_{\Re_{+}^{n}} \mathbf{0}$. A possible extension is to allow for problems where $\nabla \mathbf{f}_{0} \geq_{\mathcal{K}} \mathbf{0}$ for a more general cone $\mathcal{K}$, and compensate for this with tougher requirements on the constraint functions.

\section{REFERENCES}

Bertsekas, D. and Tsitsiklis, J. (1997). Parallel and Distributed Computation: Numerical Methods. Athena Scientific.

Boche, H. and Schubert, M. (2008). The structure of general interference functions and applications. IEEE Transactions on Information Theory, 54(11), 4980 4990.

Boyd, S. (2010). Distributed Optimization and Statistical Learning via the Alternating Direction Method of Multipliers. Foundations and Trends in Machine Learning, 3(1), 1-122.

Boyd, S., Kim, S.J., Vandenberghe, L., and Hassibi, A. (2007). A tutorial on geometric programming. Optimization and Engineering, 8(1), 67-127.

Chiuso, A., Fagnani, F., Schenato, L., and Zampieri, S. (2011). Gossip algorithms for distributed ranking. In American Control Conference (ACC), 2011, 5468-5473.

Fischione, C. (2011). Fast-lipschitz optimization with wireless sensor networks applications. IEEE Transactions on Automatic Control, 56(10), 2319-2331.

Foschini, G. and Miljanic, Z. (1993). A simple distributed autonomous power control algorithm and its convergence. Vehicular Technology, IEEE Transactions on, 42(4), 641-646.

Nedic, A., Ozdaglar, A., and Parrilo, P. (2010). Constrained Consensus and Optimization in Multi-Agent Networks. IEEE Transactions on Automatic Control, $55(4), 922-938$.

Nedic, A. and Ozdaglar, A. (2009). Distributed subgradient methods for multi-agent optimization. IEEE Transactions on Automatic Control, 54(1), 48-61.

Notarstefano, G. and Bullo, F. (2009). Distributed abstract optimization via constraints consensus: Theory and applications. IEEE Transactions on Automatic Control, (99), 1-1.

Olshevsky, A. and Tsitsiklis, J. (2009). Convergence speed in distributed consensus and averaging. SIAM Journal on Control and Optmization, 48(1), 33-55.

Rantzer, A. (2009). Dynamic dual decomposition for distributed control. American Control Conference (ACC), 2009., 884-888.

Sundhar Ram, S., Nedic, A., and Veeravalli, V. (2010). Distributed stochastic subgradient projection algorithms for convex optimization. Journal of Optimization Theory and Applications, 147, 516-545.

Sung, C. and Leung, K. (2005). A generalized framework for distributed power control in wireless networks. IEEE Transactions on Information Theory, 51(7), 2625-2635.

Wei, E., Ozdaglar, A., and Jadbabaie, A. (2011). A distributed newton method for network utility maximization, i: Algorithm. LIDS report 2832.

Yates, R. (1995). A framework for uplink power control in cellular radio systems. IEEE Journal on Selected Areas in Communications, 13(7), $1341-1347$.

Zanella, F., Varagnolo, D., Cenedese, A., Pillonetto, G., and Schenato, L. (2011). Newton-raphson consensus for distributed convex optimization. In IEEE Conference on Decision and Control (CDC), 2011. 